\title{
“A RANDOMIZED TRIAL OF STANDARD VERSUS INTENSIVE BLOOD PRESSURE CONTROL"- THE SPRINT TRIAL
}

\author{
"A RANDOMIZED TRIAL OF STANDARD VERSUS INTENSIVE BLOOD \\ PRESSURE CONTROL"- THE SPRINT TRIAL
}

Bernardo Augusto Rosário1, Bruna Loise Mayer1, Maurício de Carvalho1

\section{RESUMO}

Apesar da hipertensão arterial sistêmica ser uma condição muito prevalente, a meta pressórica a ser atingida ainda permanece incerta. O estudo SPRINT comparou o controle pressórico mais intensivo (pressão sistólica menor que $120 \mathrm{mmHg}$ ) versus o controle habitual (pressão sistólica menor que $140 \mathrm{mmHg}$ ). Neste trabalho, houve redução significativa de eventos cerebro e cardiovasculares e também de mortalidade no grupo de tratamento intensivo. Entretanto, esta redução foi associada a maior incidência de eventos adversos.

Descritores: Pressão arterial. Eventos cardiovasculares. Prevenção primária.

\section{ABSTRACT}

Despite the high prevalence of hypertension, the target blood-pressure levels to be achieved with therapy remains uncertain. The SPRINT trial compared more intensive blood pressure control (systolic blood pressure less than $120 \mathrm{mmHg}$ ) versus usual control (systolic blood pressure less than $140 \mathrm{mmHg}$ ). In this study, there was a significant reduction in cerebro- and cardiovascular events and also a reduced mortality in the intensive treated group. However, this reduction was associated with a higher incidence of adverse events.

Keywords: Blood-pressure. Cardiovascular events. Primary prevention. 


\section{INTRODUÇÃO}

A hipertensão arterial sistêmica (HAS) é uma das doenças mais prevalentes do mundo, afetando cerca de 1 bilhão de pessoas. Entre os maiores de 50 anos, a causa mais comum de HAS é a hipertensão sistólica isolada, tornando-se mais importante que a pressão diastólica. É considerada fator de risco independente para insuficiência cardíaca, acidente vascular encefálico (AVE), coronariopatia e doença renal crônica terminal.

Através de estudos observacionais, notou-se um aumento no risco cardiovascular quando a pressão arterial sistólica (PAS) ultrapassa $115 \mathrm{mmHg}$. Porém, a meta de redução de PAS ainda é incerta. Segundo recomendações do Eighth Joint National Committee (JNC 8) americano, publicado no final de 2013, niveis tensionais abaixo de 140/90 mmHg são satisfatórios para a maior parte da população. Em pacientes com 60 anos ou mais, metas pressóricas mais liberais $(<150 / 90 \mathrm{mmHg}$ ) podem ser adequadas.

O estudo SPRINT (Systolic Blood Pressure Intervention Trial), publicado no New England Journal of Medicine em novembro de 2015, objetivou avaliar os efeitos do controle rigoroso da PAS (<120 mmHg), em comparação ao controle padrão ( $<140 \mathrm{mmHg}$ ), em termos de desfechos cardiovasculares e cerebrovasculares, fatais ou não.

\section{METODOLOGIA}

Trata-se de um estudo aberto, randomizado, controlado, prospectivo, realizado em 102 estabelecimentos clínicos nos Estados Unidos e em Porto Rico, com duração prevista de cinco anos. Foram selecionados 9361 participantes acima de 50 anos de idade, com PAS entre 130 e $180 \mathrm{mmHg}$ e de alto risco cardiovascular (mortalidade em 10 anos $>15 \%$ de acordo com o score de Framingham, taxa de filtração glomerular entre 20 e $60 \mathrm{~mL} / \mathrm{min} / 1,73 \mathrm{~m}^{2}$ e/ou doença cardiovascular clínica ou subclínica). Foram excluídos aqueles que apresentavam diabetes melito (DM) ou história pregressa de AVE.

Os participantes foram randomizados em 2 grupos, sendo um submetido ao tratamento padrão (4683 participantes), que objetivou uma PAS $<140 \mathrm{mmHg}$, e o outro submetido ao tratamento intensivo (4678 participantes), que objetivou uma PAS $<120 \mathrm{mmHg}$. Todas as grandes classes de anti-hipertensivos foram incluídas no estudo e fornecidas aos participantes sem custos. Foi permitido aos investigadores a prescrição de qualquer medicamento anti-hipertensivo. 0 estudo foi financiado pelo Instituto Nacional de Saúde Americano (NIH).

\section{RESULTADOS}

As médias de PAS durante o estudo foram de $121,5 \mathrm{mmHg}$ no grupo submetido ao tratamento intensivo, e de $134,6 \mathrm{mmHg}$ no grupo submetido ao tratamento padrão (Gráfico 1). Para atingir esses níveis pressóricos, foram necessários em média 2,8 medicamentos para o grupo intensivo e 1,8 medicamentos para o grupo padrão.

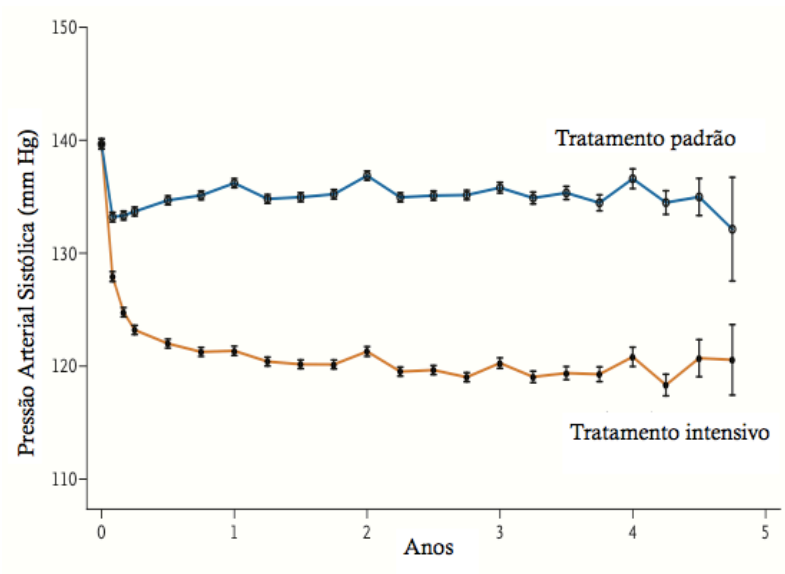

Gráfico 1. Médias de PAS ao decorrer do estudo, entre os grupos submetidos ao tratamento padrão (linha azul) e intensivo (linha alaranjada). Adaptado de referência 1.

Os resultados desse estudo demonstraram uma redução significativa no número de desfechos primários (cardiovasculares e cerebrovasculares) no grupo submetido ao tratamento intensivo, conforme exposto no gráfico abaixo (Gráfico 2). 0 desfecho primário ocorreu em $5,2 \%$ nos pacientes com tratamento intensivo contra $6,8 \%$ nos pacientes com tratamento padrão $(p<0,001)$.

o estudo também demonstrou que, em comparação ao grupo submetido ao tratamento padrão, os pacientes do grupo tratamento intensivo apresentaram mais eventos adversos, como hipotensão, síncope, distúrbios hidroeletrolíticos (notadamente hipocalemia e hiponatremia) e injúria renal aguda. Curiosamente, os participantes submetidos ao tratamento intensivo apresentaram menor índice de hipotensão postural isolada, se comparados aos submetidos ao tratamento padrão.

\section{DISCUSSÃO}

Os resultados foram muito positivos em pacientes com tratamento intensivo. De fato, cerca 61 pacientes tiveram de ser tratados por 3,3 anos (NNT), para evitar um evento cardiovascular ou AVE.

O estudo SPRINT englobou uma amostra representativa de participantes, inclusive com proporção considerável de pacientes idosos, o que aumenta sua validade externa. Além disso, as metas de PAS foram 
atingidas em grande parte dos participantes, preocupação mencionada pelos autores.

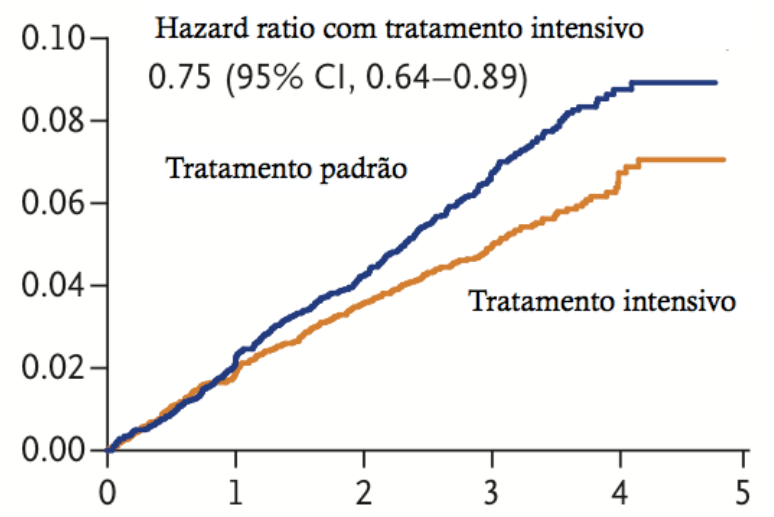

Gráfico 2. Número de desfechos primários no grupo submetido ao tratamento intensivo, em comparação ao grupo submetido ao tratamento padrão (risco relativo 0,75; IC 95\%, 0,64-0,89; $\mathrm{p}<0,001)$. Adaptado de referência 1.

Um achado relevante (e preocupante) do estudo foi o maior índice de insuficiência renal aguda no grupo de participantes submetido ao tratamento intensivo. Diversas hipóteses para esse fato foram levantadas. Dentre elas, o efeito hemodinâmico intra-renal causado pela maior redução da PA (esse efeito possivelmente reversível) e o maior uso de medicamentos que contribuiriam para a redução da taxa de filtração glomerular, como os inibidores da enzima conversora de angiotensina (IECA), bloqueadores do receptor de angiotensina II (BRA-II) e diuréticos. Ressalta-se no artigo que, até o momento, não há evidência de injúria renal permanente ao se buscar metas mais baixas de PA.

O estudo apresenta algumas limitações, como a exclusão de pacientes diabéticos, com AVE prévio, de indivíduos mais jovens (abaixo de 50 anos) e de idosos institucionalizados. Pacientes com hipertensão severa (PAS acima de $180 \mathrm{mmHg}$ ) também foram excluídos do estudo. Outro fator limitante reside no fato que, de maneira geral, nos consultórios médicos dos EUA, a PA menor que 140/90 mmHg (meta padrão) só é atingida em 50\% dos pacientes, o que tornaria o controle agressivo um desafio ainda maior. Por último, ainda permanece uma incógnita se o custo de usar mais medicamentos, realizar mais consultas de acompanhamento e exames de rotina para se atingir o controle pressórico desejado pelos autores é factível pelos sistemas de saúde ao redor do mundo.

\section{CONCLUSÃO}

A partir do exposto acima, conclui-se que o controle rigoroso da PAS (abaixo de $120 \mathrm{mmHg}$ ), em pacientes de alto risco cardiovascular, sem DM ou AVE prévio, tende a reduzir eventos cardiovasculares e cerebrovasculares, porém às custas de um maior número de eventos adversos. De modo pragmático, as abordagens devem ser individualizadas e implementadas em uma relação médico-paciente bem estabelecida.

\section{REFERÊNCIAS}

1. The SPRINT Research Group. A randomized trial of intensive versus standard blood-pressure control. The New England Journal of Medicine 2015 Nov; [Epub ahead of print] http://www.nejm.org/doi/full/10.1056/NEJMoa1511 939?query=featured_home\&\#t=article. 